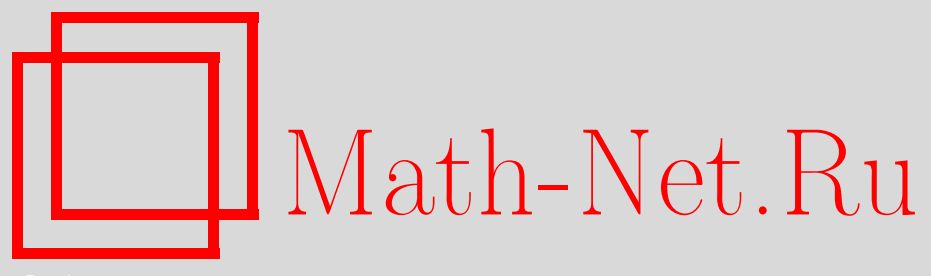

Д. М. Спайт, Преломление магнитных пузырей и квазибризеры в неоднородных антиферромагнетиках, $T M \Phi$, 2007, том 152, номер 1, 191-208

DOI: https://doi.org/10.4213/tmf6080

Использование Общероссийского математического портала Math-Net.Ru подразумевает, что вы прочитали и согласны с пользовательским соглашением http://www.mathnet.ru/rus/agreement

Параметры загрузки:

IP: 3.95 .254 .165

26 апреля 2023 г., 09:33:34

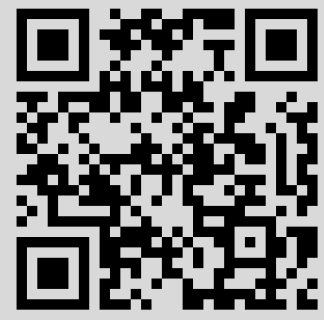




\title{
ПРЕЛОМЛЕНИЕ МАГНИТНЫХ ПУЗЫРЕЙ И КВАЗИБРИЗЕРЫ В НЕОДНОРОДНЫХ АНТИФЕРРОМАГНЕТИКАХ
}

\begin{abstract}
Исследована динамика солитонов в виде магнитных пузырей в двумерной изотропной антиферромагнитной спиновой решетке в случае, когда обменный интеграл $J(x, y)$ зависит от координат. В почти непрерывном режиме эта система описывается релятивистской $O(3)$ сигма-моделью в пространстве-времени с определяемой $J$ пространственно неоднородной метрикой. Для описания низкоэнергетической солитонной динамики в данной системе используется геодезическая аппроксимация: движение $n$-солитона аппроксимируется геодезическим движением в пространстве модулей $\mathrm{M}_{n}$ статических $n$-солитонов, снабженном $L^{2}$-метрикой $\gamma$. Получены явные формулы для $\gamma$ при различных естественных выборах $J(x, y)$. Из них следует, что траектории отдельных солитонов испытывают преломление, а $J^{-1}$ аналогичен показателю преломления, и что такой эффект преломления позволяет конструировать простые пузырьковые линзы и "пузырьководы." Подробно рассмотрен случай, когда $J$ имеет дисковую неоднородность (со значением $J_{+}$снаружи диска и $J_{-}<J_{+}$внутри него). Приводятся аргументы в пользу того, что при достаточно больших значениях отношения $J_{+} / J_{-}$этот тип антиферромагнетиков поддерживает приближенные квазибризеры: два или более совпадающих пузыря, заключенных внутри диска, испытывают внутреннее вращение, в то время как форма этих пузырей испытывает периодические колебания с несоизмеримыми в случае общего положения периодами.
\end{abstract}

Ключевые слова: топологические солитоны, геодезическая аппроксимация, антиферромагнетик Гейзенберга.

\section{1. ВВЕДЕНИЕ}

Целью настоящей работы является анализ динамики пузыреподобных топологических солитонов, возникающих в двумерном классическом антиферромагнетике

${ }^{*}$ School of Mathematics, University of Leeds, Leeds LS2 9JT, UK. E-mail: speight@maths.leeds.ac.uk 
Гейзенберга, обменное взаимодействие в котором является изотропным, но неоднородным. В каждом узле $(i, j) \in \mathbb{Z}^{2}$ квадратной решетки имеется вектор классического спина $\mathbf{S}_{i j} \in \mathbb{R}^{3},\left|\mathbf{S}_{i j}\right|^{2}=s^{2}$, эволюционирующий согласно закону

$$
\frac{d \mathbf{S}_{i j}}{d \tau}=-\mathbf{S}_{i j} \times \frac{\partial H}{\partial \mathbf{S}_{i j}}, \quad H:=\sum_{i, j} J_{i j}\left[2 s^{2}+\mathbf{S}_{i j} \cdot\left(\mathbf{S}_{i, j+1}+\mathbf{S}_{i+1, j}\right)\right],
$$

где $\tau$ - время, а $J_{i j}$ - положительные константы. Это обычный плоский изотропный антиферромагнетик Гейзенберга, но в случае, когда обменный интеграл $J$ выбран неоднородным, т.е. зависящим от координат. Экспериментальные способы достижения такой $J$-неоднородности при этом не фиксируются. Предложение (возможно, наивное) заключается в том, что образец одного антиферромагнитного материала местами мог бы быть обогащен атомами различных антиферромагнитных сортов. Заметим, что поскольку $J_{i j}>0$ для всех $i, j$, величина $H$ минимизируется, когда каждый спин, как и в однородном случае, направлен противоположно своим ближайшим соседям.

Давно известно [1], что динамика однородной спиновой решетки (с постоянной $\left.J_{i j}=J>0\right)$ описывается в непрерывном пределе с помощью нелинейной $O(3)$ сигма-модели. В работе [2] с помощью модифицированного процесса димеризации Коминеаса и Папаниколау [3] было показано, что этот результат естественным образом обобщается на неоднородную задачу, рассматриваемую в настоящей работе. Другими словами, непрерывный предел системы (1) описывается нелинейным дифференциальным уравнением в частных производных (ДУЧП)

$$
\mathbf{n} \times \mathcal{D} \mathbf{n}=\mathbf{0}, \quad \mathcal{D} \mathbf{n}:=\frac{\partial^{2} \mathbf{n}}{\partial t^{2}}-J(x, y)^{2}\left(\frac{\partial^{2} \mathbf{n}}{\partial x^{2}}+\frac{\partial^{2} \mathbf{n}}{\partial y^{2}}\right),
$$

где $\mathbf{n}: \mathbb{R}^{2+1} \rightarrow \mathbb{R}^{3}$ имеет норму $|\mathbf{n}|=1, t$ - перемасштабированная временна́я переменная, а $J(x, y)$ - непрерывное приближение величины $J_{i j}$. Соотношение между $\mathbf{n}$ и $\mathbf{S}_{i j}$ является деликатным. Если выбрать постоянную решетки $\varepsilon>0$ малой, то связанная с решением $\mathbf{n}(t, x, y)$ уравнения (2) спиновая динамика будет иметь вид

$$
\mathbf{S}_{i j}(\tau)=(-1)^{i-j} s \mathbf{n}(2 \sqrt{2} s \varepsilon \tau, i \varepsilon, j \varepsilon)+O(\varepsilon) .
$$

Грубо говоря, следует представлять себе поле $\mathbf{n}$ "сидящим" на квадратной решетке с постоянной $\varepsilon$, разбитой на черные и белые подрешетки, как шахматная доска. Белые спины эволюционируют как $s \mathbf{n}(t)$, а черные - как $-s \mathbf{n}(t)$, где $t=2 \sqrt{2} s \varepsilon \tau$.

Уравнение (2) все еще является полевым уравнением нелинейной $O(3)$ сигмамодели на $\mathbb{R}^{2+1}$, но теперь пространство-время снабжено пространственно неоднородной лоренцевой метрикой

$$
\eta=d t^{2}-\frac{1}{J(x, y)^{2}}\left(d x^{2}+d y^{2}\right)
$$

Заметим, что статическое полевое уравнение не зависит от $J$, так что обычные бугры Белавина-Полякова для однородной системы [4], которые мы в данном контексте 
будем называть пузырями (по аналогии с магнитными пузырями в ферромагнетиках), переносятся без изменений на неоднородную систему. С учетом релятивистской природы уравнения (2) эти статические пузыри можно немедленно подвергнуть бусту, что даст решения в виде движущихся пузырей. Однако из-за неоднородности метрики $\eta$ такие пузыри не обязательно движутся вдоль прямых линий. Детальное исследование траектории одиночных пузырей, взаимодействующих с различными $J$-неоднородностями, было представлено в работе [2]. Основное заключение состоит в том, что в подавляющем числе случаев траектории пузырей испытывают преломление по аналогии с геометрической оптикой, причем $J(x, y)^{-1}$ играет роль показателя преломления среды. Углы падения и выхода пузыря, пересекающего доме́нную стенку, например, J-неоднородность, связаны законом Снеллиуса, причем при достаточно большом угле наклона падения происходит полное внутреннее отражение.

В настоящей работе мы дадим краткий обзор описанного ключевого явления преломления, затем перейдем к изучению некоторых новых динамических задач, которые не рассматривались в работе [2]. Преломление пузырей можно вывести, используя только законы сохранения и допущение о том, что рассеяние пузыря на доме́нной стенке приближенно можно считать упругим [2]. Однако в настоящей работе мы будем проводить наши рассуждения относительно динамики пузырей полностью в рамках геодезической (или адиабатической) аппроксимации Мантона [5], представляющей собой стандартный подход к низкоэнергетической солитонной динамике в теориях поля такого типа. В разделе 2 построена геодезическая аппроксимация применительно к динамике медленных пузырей. Далее в разделе 3 этот подход используется для изучения взаимодействия одиночного пузыря с доме́нной стенкой, а также с $J$-неоднородностями типа корыта и дисковыми $J$-неоднородностями. Наконец, в разделе 4 изучается вращательно-эквивариантная динамика $n$ совпадающих пузырей в дисковой $J$-неоднородности. Мы получаем динамические явления, полностью отсутствующие в однородной модели и не имеющие аналогов в оптике. Форма пузыря с зарядом $n$, приведенного во вращение вокруг своей оси симметрии, может в некоторых случаях претерпевать периодические колебания. Эти (приближенные) решения пространственно локализованы, а поскольку период осцилляций формы не обязан, вообще говоря, быть соизмеримым с периодом внутреннего вращения, они оказываются и квазипериодичными по времени. Таким образом, эти решения представляют собой квазибризеры. В однородной модели, напротив, приведенный во вращение пузырь с зарядом $n$ просто расширяется до бесконечности. Неоднородность $J$ может, таким образом, оказывать стабилизирующее влияние на вращающиеся пузыри.

\section{2. ГЕОДЕЗИЧЕСКАЯ АППРОКСИМАЦИЯ}

Уравнение (2) представляет собой вариационное уравнение, для которого действие имеет вид

$$
S=\frac{1}{2} \int d t d x d y \sqrt{|\eta|} \partial_{\mu} \mathbf{n} \cdot \partial_{\nu} \mathbf{n} \eta^{\mu \nu}=\int d t(T-V),
$$


где величины

$$
T=\frac{1}{2} \int d x d y \frac{1}{J(x, y)^{2}}\left|\frac{\partial \mathbf{n}}{\partial t}\right|^{2}, \quad V=\frac{1}{2} \int d x d y\left(\left|\frac{\partial \mathbf{n}}{\partial x}\right|^{2}+\left|\frac{\partial \mathbf{n}}{\partial y}\right|^{2}\right)
$$

отождествляются с функционалами кинетической и потенциальной энергии, соответственно. Заметим, что $T$ зависит от $J$, но $V$ от него не зависит. Это является следствием того важного факта, что потенциальная энергия сигма-модели (часто называемая гармоническим отображением, или энергией Дирихле) на пространстве размерности 2 зависит только от конформного класса пространственной метрики. В нашей задаче эта метрика равна $g=J(x, y)^{-2}\left(d x^{2}+d y^{2}\right)$ и конформно-эквивалентна евклидовой метрике при любом выборе $J(x, y)$. Отсюда следует, что анализ, проведенный Белавиным и Поляковым для статической однородной модели [4], не изменяется и для неоднородной системы. Имеется топологическое нижнее ограничение для энергии

$$
V[\mathbf{n}] \geqslant 4 \pi|n|,
$$

где $n$ - топологическая степень отображения $\mathbf{n}: \mathbb{R}^{2} \cup\{\infty\} \rightarrow S^{2}$. Полезно определить комплексные координаты $z=x+i y$ на пространственной плоскости и $u=$ $\left(n_{1}+i n_{2}\right) /\left(1-n_{3}\right)$ на таргет-сфере (последняя является образом вектора $\mathbf{n}$ при стереографической проекции из $(0,0,1)$ в экваториальную плоскость). Тогда при $n \geqslant 0$ определенная в (7) граница достигается, если и только если $u(z)$ является рациональным отображением алгебраической степени $n$, т.е.

$$
u(z)=\frac{a_{0}+a_{1} z+\cdots+a_{n} z^{n}}{b_{0}+b_{1} z+\cdots+b_{n} z^{n}}
$$

где $a_{i}, b_{i}$ - комплексные постоянные, по крайней мере одна из величин $a_{n}, b_{n}$ не равна нулю, а числитель и знаменатель не имеют общих корней. Поскольку такие отображения глобально минимизируют $V$ внутри своего гомотопического класса, они автоматически являются устойчивыми статическими решениями модели.

Без потери общности можно выбрать граничные значения для $\mathbf{n}$ на пространственной бесконечности в виде $(0,0,1)$, т.е. $u(\infty)=\infty$, так что $b_{n}=0$, а потому $a_{n} \neq 0$. Поскольку $a_{n} \neq 0$, можно разделить числитель и знаменатель на $a_{n}$ и переименовать $a_{i} / a_{n} \mapsto a_{i}, b_{i} / b_{n} \mapsto b_{i}$, так что общим статическим решением будет

$$
u(z)=\frac{a_{0}+a_{1} z+\cdots+a_{n-1} z^{n-1}+z^{n}}{b_{0}+b_{1} z+\cdots+b_{n-1} z^{n-1}} .
$$

Это решение единственным образом определяется $2 n$ комплексными константами $a_{0}, \ldots, b_{n-1}$. Поэтому пространство модулей (или пространство параметров) $\mathrm{M}_{n}$ статических решений степени $n$ отождествляется с открытым подмножеством в $\mathbb{C}^{2 n}$, а именно с дополнением к алгебраическому многообразию комплексной коразмерности 1 , на котором числитель и знаменатель имеют общие корни. Например, для пузыря общего вида со степенью 1 имеем

$$
u(z)=\chi^{-1} e^{-i \psi}(z-w)
$$


где $\chi \in(0, \infty), \psi \in[0,2 \pi]$ и $w=w_{1}+i w_{2} \in \mathbb{C}-$ константы, интерпретируемые соответственно как ширина, внутренняя фаза и координата пузыря. Тогда $\mathrm{M}_{1}$ диффеоморфно $\mathbb{C}^{\times} \times \mathbb{C}$, где $\mathbb{C}^{\times}=\mathbb{C} \backslash\{0\}$. В общем случае $\mathrm{M}_{n}$ имеет вещественную размерность $4 n$, и его можно рассматривать как конфигурационное пространство $n$ единичных пузырей. Однако оно не диффеоморфно $\left(\mathrm{M}_{1}\right)^{n}$, и глобально его параметры нельзя отождествить с координатами, ширинами и ориентациями $n$ отдельных пузырей.

Следуя работам [6], [7], можно исследовать низкоэнергетическую динамику $n$ пузырей в рамках геодезической аппроксимации Мантона [5]. Идея состоит в том, что $4 n$-мерное пространство $\mathrm{M}_{n}$ статических решений степени $n$ представляет собой плоское дно долины в пространстве всех отображений степени $n, \mathbb{R}^{2} \rightarrow S^{2}$, на котором $V$ достигает своего минимального значения, равного $4 \pi n$. Рассмотрим задачу Коши, в которой пузыри, составляющие статический $n$-пузырь, приведены в относительное движение с малыми скоростями. Тогда исходное поле лежит в $\mathrm{M}_{n}$, исходное поле скоростей является касательным к $\mathrm{M}_{n}$, и система имеет только небольшое количество кинетической энергии. Поэтому из-за сохранения энергии $E=T+V$ последующее движение системы в полевом конфигурационном пространстве ограничено областью вблизи $\mathrm{M}_{n}$ (заметим, что $T$ строго положительно). Это подсказывает разумность аппроксимации с помощью коллективных координат там, где движение заключено в $\mathrm{M}_{n}$ во все моменты времени. Эту аппроксимацию часто называют адиабатической, поскольку предполагается, что в любой момент времени полевая конфигурация хорошо аппроксимируется статическим решением, однако параметры этого решения могут медленно меняться со временем.

Пусть $q^{1}, \ldots, q^{4 n}$ - произвольная вещественная система координат на $\mathrm{M}_{n}$ (например, вещественная и мнимая части $\left.a_{0}, \ldots, a_{n-1}, b_{0}, \ldots, b_{n-1}\right)$. Обозначим через $u_{0}(z ; q)$ статический $n$-пузырь, соответствующий $q=\left(q^{1}, \ldots, q^{4 n}\right)$. Тогда согласно адиабатической аппроксимации имеем

$$
u(z, t)=u_{0}(z ; q(t))
$$

Теперь подставим (11) в выражение для $S$ и получим вариационные уравнения для $q$. Заметим, что $V \equiv 4 \pi n$ (константа) для всех полей вида (11), а $T$ квадратично по производным по времени, так что действие сводится к (использовано эйнштейновское соглашение о суммировании)

$$
S=\frac{1}{2} \int d t\left(\gamma_{i j} \dot{q}^{i} \dot{q}^{j}-4 \pi n\right), \quad \gamma_{i j}(q):=\int \frac{d x d y}{J(x, y)^{2}} \frac{4}{\left(1+\left|u_{0}(z ; q)\right|^{2}\right)^{2}} \frac{\partial u_{0}}{\partial q^{i}} \frac{\partial \bar{u}_{0}}{\partial q^{j}}
$$

Это выражение представляет собой геодезическое движение на $\mathrm{M}_{n}$ с метрикой $\gamma=$ $\gamma_{i j}(q) d q^{i} d q^{j}$. Поэтому мы ожидаем, что $u(z, t)$ будет хорошо аппроксимироваться геодезическим движением в $\left(\mathrm{M}_{n}, \gamma\right)$, по крайней мере, при низких скоростях. Следует подчеркнуть: это не означает, что отдельные пузыри следуют геодезическим в физическом пространстве $\left(\mathbb{R}^{2}, g\right)$ даже в случае $n=1$. Действительно, движение 2-пузырей даже в евклидовом пространстве (т.е. в однородной модели) в сильной 
степени нетривиально [6], [7]. Например, два тождественных пузыря, непосредственно налетающие друг на друга, не проходят друг через друга неизменными, но и не сближаются до некоторого минимального расстояния, чтобы потом удалиться друг от друга (как можно было бы подумать) вдоль тех линий, по которым они пришли. Вместо этого они сливаются, образуя кольцеподобный 2-пузырь, а затем разрываются на части и удаляются вдоль линии, перпендикулярной линии их прихода. Такое поведение носит общий характер для планарных топологических солитонов в релятивистских теориях поля; оно хорошо понято в рамках геодезической аппроксимации [8]. Концептуальные рамки и обоснованность геодезической аппроксимации подробно обсуждаются в работе [9].

Метрика $\gamma$, а потому и динамика, сильно зависят от $J(x, y)$. Основная задача такого подхода к топологической солитонной динамике состоит в вычислении этой метрики. Даже в случае степени 1 ее невозможно вычислить явно, если только $J$ не имеет очень простого симметричного вида. Тем не менее можно получить много качественной информации относительно $\gamma$ и прийти к хорошему пониманию динамики отдельного пузыря даже в отсутствие явных формул. В случае более высокой степени $n \geqslant 2$ пространство модулей слишком велико для того чтобы можно было рассматривать общую геодезическую задачу, даже если метрика $\gamma$ известна явно. Для дальнейшего нам необходимо редуцировать размерность, полностью отождествив геодезические подмногообразия. Это равнозначно наложению симметрийных связей на исходные данные.

В случае $n=1$ метрика имеет очень простой вид. Если $J$ постоянна, то мы имеем дело со стандартной сигма-моделью, и можно найти, что величины $\chi$ и $\psi$ замораживаются вследствие бесконечной инерции $\left(\gamma_{\chi \chi}=\gamma_{\psi \psi}=\infty\right)$, a $\gamma=4 \pi J^{-2} d w d \bar{w}$, так что пузыри просто движутся с постоянной скоростью [6]. В другом предельном случае $J(x, y)=1+x^{2}+y^{2}$ мы получаем сигма-модель на круглой 2-сфере, и в этом случае динамика пузырей более разнообразна [10]. При таком выборе значение $J$ не ограничено, что, впрочем, представляется нефизичным. Предполагая, что $J$ остается ограниченным, получаем, что величины $\chi$ и $\psi$ замораживаются в силу аргументов, в основном идентичных аргументам Уорда. Без потери общности можно положить $\psi=0$. Ширина $\chi$ остается свободным, но замороженным параметром. Метрика на $M_{1}^{\chi}$ (на листе пространства $\mathrm{M}_{1}$ с шириной $\chi$ и фазой 0 ) тогда принимает вид

$$
\gamma=f_{\chi}(w)\left(d w_{1}^{2}+d w_{2}^{2}\right)
$$

где

$$
f_{\chi}(w)=\int \frac{d z d \bar{z}}{J(z, \bar{z})^{2}} \frac{4 \chi^{-2}}{\left(1+\chi^{-2}|z-w|^{2}\right)^{2}}=\int \frac{4 d u d \bar{u}}{\left(1+|u|^{2}\right)^{2}} \frac{1}{J(\chi u+w)^{2}}
$$

- интеграл от $J(\chi u+w)^{-2}$ по стандартной единичной сфере, на которой $u$ является стереографической координатой. Заметим, что

$$
\lim _{\chi \rightarrow 0} \gamma=\frac{4 \pi}{J(w)^{2}}\left(d w_{1}^{2}+d w_{2}^{2}\right)=4 \pi g .
$$


Поэтому в пределе нулевой ширины пузырей траектории изолированных пузырей стремятся к геодезическим не только в $\mathrm{M}_{1}^{\chi}$, но и в физической плоскости. Из-за того, что ядро пузырей имеет конечный размер, можно рассматривать метрику $\gamma$ на $\mathrm{M}_{1}^{\chi}$ как "размазанный” вариант метрики $g$.

\section{3. ПРЕЛОМЛЕНИЕ ПУЗЫРЕЙ}

Простейшая из всех $J$-неоднородностей - это доме́нная стенка. Предположим, что $J$ зависит только от $x$, его значение постоянно вне небольшой окрестности $(-\delta, \delta)$ точки $x=0$ и монотонно возрастает от $J_{-}$до $J_{+}$, когда $x$ проходит через эту окрестность. Появление обменного интеграла $J(x)$ такого вида возможно, если, например, один конец антиферромагнетика обогащен, а другой - нет. Из выражения (14) немедленно следует, что конформный множитель $f_{\chi}(w)$ метрики $\gamma$ на $\mathrm{M}_{1}^{\chi}$ зависит только от $w_{1}$, монотонно убывает и имеет предел

$$
\lim _{w_{1} \rightarrow \pm \infty} f_{\chi}\left(w_{1}\right)=\frac{4 \pi}{J_{ \pm}^{2}} .
$$

В нашем анализе взаимодействия пузыря с доме́нной стенкой мы будем использовать только эти свойства $f_{\chi}$. Явную формулу для $f_{\chi}$ можно получить в пределе, когда ширина $\delta \rightarrow 0$ доме́нной стенки обращается в нуль. Если "идеализировать" доме́нную стенку, описав ее ступенчатой функцией

$$
J(x)= \begin{cases}J_{+}, & x \geqslant 0 \\ J_{-}, & x<0\end{cases}
$$

то из выражения (14) с помощью несложного упражнения в сферической геометрии [2] можно найти, что

$$
f_{\chi}(a)=\frac{2 \pi}{J_{-}^{2}}\left[1-\frac{a_{1}}{\sqrt{\chi^{2}+a_{1}^{2}}}\right]+\frac{2 \pi}{J_{+}^{2}}\left[1+\frac{a_{1}}{\sqrt{\chi^{2}+a_{1}^{2}}}\right] .
$$

Заметим, что, как и предполагалось, $f_{\chi}$ гладко убывает от $4 \pi / J_{-}^{2}$ до $4 \pi / J_{+}^{2}$.

Для анализа геодезического потока в $\mathrm{M}_{1}^{\chi}$ полезно использовать гамильтонов формализм. Координаты $w_{1}, w_{2}$ имеют канонически-сопряженные импульсы

$$
p_{1}=f\left(w_{1}\right) \dot{w}_{1}, \quad p_{2}=f\left(w_{1}\right) \dot{w}_{2},
$$

при этом поток порождается гамильтонианом

$$
H=\frac{1}{2 f\left(w_{1}\right)}\left(p_{1}^{2}+p_{2}^{2}\right) .
$$

Как $H$, так и $p_{2}$ представляют собой интегралы движения. Поскольку геодезические траектории не зависят от начальной скорости, без потери общности можно считать, что $H=1 / 2$. Тогда траектории попадают в однопараметрическое семейство, занумерованное сохраняющимся импульсом $p_{2}$, который с учетом $(20)$ должен 


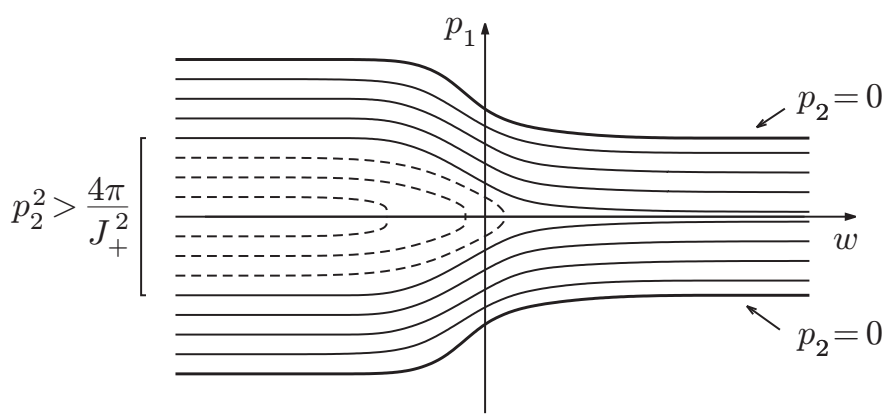

Рис. 1. Спроектированный фазовый портрет геодезического потока в $\mathrm{M}_{1}^{\chi}$ для $J$-неоднородности в виде доме́нной стенки. Обменный интеграл $J_{+}$справа от стены превосходит обменный интеграл $J_{-}$слева от нее. Траектории заключены между верхней и нижней жирными кривыми, для которых $p_{2}=0$. Штриховые траектории, для которых выполнено $4 \pi / J_{+}^{2}<p_{2}^{2}<4 \pi / J_{-}^{2}$, соответствуют пузырям, испытывающим полное внутреннее отражение.

удовлетворять соотношению $p_{2}^{2}<\sup _{w_{1} \in \mathbb{R}} f\left(w_{1}\right)=4 \pi / J_{-}^{2}$. Образ траектории с импульсом $p_{2}$ при проекции на плоскость $\left(w_{1}, p_{1}\right)$ в фазовом пространстве лежит на множестве постоянного значения $f\left(w_{1}\right)-p_{1}^{2}=p_{2}^{2}$. Схематическое изображение этих (спроектированных) траекторий представлено на рис. 1. Они заключены в области, имеющей форму бутылки и ограниченной кривыми $p_{1}= \pm \sqrt{f\left(w_{1}\right)}$, объединение которых является множеством постоянного значения $p_{2}^{2}=0$. Сами эти кривые соответствуют пузырю, падающему на доме́нную стенку под нулевым углом (т.е. ортогонально стенке) и проходящему прямо через нее слева направо (верхняя кривая) или справа налево (нижняя кривая). Очевидно, что имеется два типа траекторий. Если $0 \leqslant p_{2}^{2}<4 \pi / J_{+}^{2}$, то $\dot{w}_{1}$ никогда не обращается в нуль, и пузырь проходит через стенку. Мы называем эти траектории преломленными. Если $4 \pi / J_{+}^{2}<p_{2}^{2}<4 \pi / J_{-}^{2}$, то для траекторий имеем $\lim _{t \rightarrow \pm \infty} w_{1}(t)=-\infty$, так что пузырь подходит к стенке слева и отражается от нее. Мы называем эти траектории отраженными. Если $\theta(t)-$ угол между направлением скорости пузыря и осью $x$, то $\dot{w}=|\dot{w}| e^{i \theta(t)}$. Тогда имеем

$$
\sin \theta(t)=\frac{\dot{w}_{2}}{\sqrt{\dot{w}_{1}^{2}+\dot{w}_{2}^{2}}}=\frac{p_{2}}{\sqrt{p_{1}^{2}+p_{2}^{2}}}=\frac{p_{2}}{\sqrt{f\left(w_{1}(t)\right)}}
$$

Поэтому для преломленной траектории при падении пузыря на доме́нную стенку слева имеем

$$
\frac{\sin \theta(\infty)}{\sin \theta(-\infty)}=\frac{f(-\infty)}{f(\infty)}=\frac{J_{+}^{-1}}{J_{-}^{-1}}
$$

а потому углы падения и выхода траектории пузыря $\theta(-\infty)$ и $\theta(\infty)$ связаны законом преломления Снеллиуса, причем $J^{-1}$ отождествляется с показателем преломления. Если $\sin \theta(-\infty)>J_{-} / J_{+}$, то $p_{2}^{2}>4 \pi / J_{+}^{2}$, и имеет место полное внутреннее отражение. 
Интересно выполнить подобный качественный анализ в случае $J$-неоднородности в виде прямого корыта. Предположим, что $J$ снова не зависит от $y ; J(x)=J_{1}$ при $|x| \geqslant 1+\delta$, где $\delta>0$ мало; $J(x)=J_{2}<J_{1}$ при $|x| \leqslant 1-\delta$; а также что $J$ монотонно интерполирует между постоянными значениями $J_{1}$ и $J_{2}$ в узких переходных интервалах $(-1-\delta,-1+\delta)$ и $(1-\delta, 1+\delta)$. С учетом закона Снеллиуса можно ожидать, что для неоднородности доме́нных стенок (22) получится "пузырьковод": имеется бесконечная полоса с показателем преломления $1 / J_{2}$ бо́льшим, чем показатель преломления $1 / J_{1}$ в окружающей области, так что пузырь, исходно находившийся в полосе и двигавшийся примерно вдоль нее, должен быть захвачен в полосу за счет полного внутреннего отражения подобно тому, как луч света удерживается внутри волоконно-оптического кабеля.

Для нас снова важно понимание того, какую роль играет конформный множитель $f_{\chi}(w)$ метрики на $\mathrm{M}_{1}^{\chi}$. Ясно, что $f_{\chi}$ зависит только от $w_{1}$ и стремится к $4 \pi / J_{1}^{2}$ при $w_{1} \rightarrow \pm \infty$. В общем случае он имеет форму бугра с единственной критической точкой - глобальным максимумом. Если предположить, что $J\left(w_{1}\right)$ является четным, то этим же свойством будет обладать и $f_{\chi}\left(w_{1}\right)$, и этот максимум, который всегда меньше $4 \pi / J_{2}^{2}$, будет достигаться при $w_{1}=0$. Как и выше, в пределе резкой стенки, $\delta \rightarrow 0$, можно найти явное выражение для $f_{\chi}\left(w_{1}\right)$. Дело в том, что в этом пределе величина $J\left(w_{1}\right)$ кусочно-постоянна, а потому, исходя из $(14)$, нам надо только вычислить площадь области на $S^{2}$, стереографический образ которой является вертикальной полосой $\{u \in \mathbb{C}:-1<\operatorname{Re}(\chi u+w)<1\}$. Это легко сделать, используя вычисления для резких доме́нных стенок, проведенные в работе [2] (которые и привели к формуле (18)). Получаем

$$
f_{\chi}\left(w_{1}\right)=\frac{4 \pi}{J_{1}^{2}}+2 \pi\left(\frac{1}{J_{2}^{2}}-\frac{1}{J_{1}^{2}}\right)\left[\frac{w_{1}+1}{\sqrt{\left(w_{1}+1\right)^{2}+\chi^{2}}}-\frac{w_{1}-1}{\sqrt{\left(w_{1}-1\right)^{2}+\chi^{2}}}\right]
$$

Заметим, что эта функция обладает предсказанными качественными свойствами.

Гамильтонов анализ геодезического потока в $\left(\mathrm{M}_{1}^{\chi}, \gamma\right)$ проводится аналогично случаю доме́нных стенок. Мы снова выбираем $H=1 / 2$ и проводим кривые постоянного уровня $f_{\chi}\left(w_{1}\right)-p_{1}^{2}=p_{2}^{2}$ на плоскости $\left(w_{1}, p_{1}\right)$ для $0 \leqslant p_{2}^{2}<f_{\max }=f_{\chi}(0)$. Как и ранее, возникают два режима, разделенные значением $p_{2}^{2}=p_{*}^{2}=4 \pi / J_{1}^{2}$ (см. рис. 2 ). При $p_{2}<p_{*}$ траектории не ограничены, и $\dot{w}_{1}$ никогда не обращается в нуль. Это соответствует картине, когда пузырь снаружи попадает в корыто и проходит через него, в процессе этого дважды испытывая преломление, так что его начальная и конечная скорости совпадают. При $p_{2}>p_{*}$ (спроектированные) траектории ограничены и являются периодическими. Они соответствуют картине, когда пузырь двигается вдоль корыта вследствие полного внутреннего отражения. Реальная траектория пузыря в физической плоскости не является, конечно, периодической, поскольку он равномерно дрейфует в $y$-направлении ( $\dot{w}_{2}$ никогда не обращается в нуль, однако это не константа). Заметим, что даже в этом режиме захвата траектория пузыря не обязательно заключена внутри самого корыта. Действительно, при любом $R>0$ существует захваченная геодезическая, для которой $\max _{t}\left|w_{1}(t)\right|>R$. В этом смыс- 


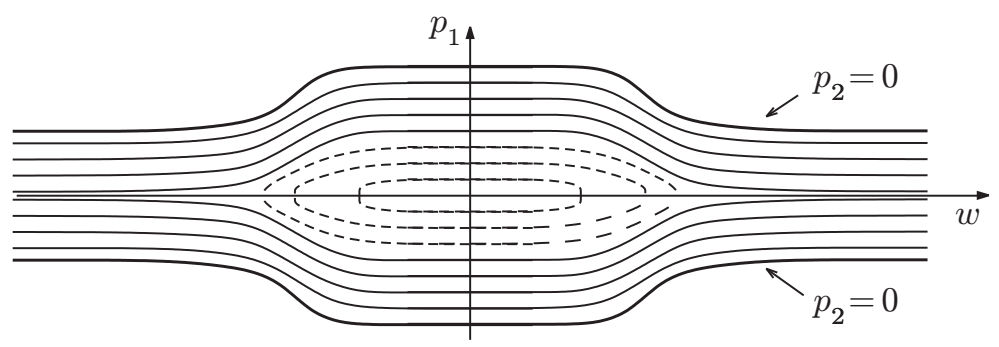

Рис. 2. Спроектированный фазовый портрет геодезического потока в $\mathrm{M}_{1}^{\chi}$ для $J$-неоднородности в виде корыта. Обменный интеграл равен $J_{1}$ за пределами вертикальной полосы, где он уменьшается до $J_{2}<J_{1}$. Замкнутые штриховые траектории, для которых $p_{2}^{2}>4 \pi / J_{1}^{2}$, соответствуют пузырям, движение которых направлено вдоль полосы за счет полного внутреннего отражения.

ле поведение траекторий пузырей заметно отличается от поведения световых лучей в геометрической оптике.

Эффект "пузырьковода" не зависит от того, является ли корыто прямым. Случай круглого корыта (т.е. такого, что область пониженных значений $J$ представляет собой кольцо) рассматривался в работе [2], где были получены качественно подобные результаты.

Другой интересный эффект, напоминающий геометрическую оптику, состоит в явлении фокусировки траектории пузырей круглой “линзой." Представим себе, что $J$ зависит только от $|z|$, так что $J(|z|)=J_{-}$при $|z| \leqslant 1-\delta, J(|z|)=J_{+}>J_{-}$ при $|z|>1+\delta$, гладко и монотонно интерполируя между этими значениями в узком кольце $1-\delta<|z|<1+\delta$. Таким образом, имеется диск, в котором показатель преломления $1 / J_{-}$больше, чем в окружающей среде, так что можно ожидать, что траектории отдельных пузырей будут преломляться этой неоднородностью подобно тому, как преломляются лучи света, проходящие через круглую линзу. Из формулы (14) немедленно следует, что конформный множитель в метрике зависит только от $|w|$. В пределе резких стенок $\delta \rightarrow 0$ можно явно вычислить

$$
\begin{aligned}
f_{\chi}(|w|)=\frac{2 \pi}{J_{-}^{2}} & {\left[1-\frac{\chi^{2}+|w|^{2}-1}{\sqrt{\chi^{4}+2 \chi^{2}\left(|w|^{2}+1\right)+\left(|w|^{2}-1\right)^{2}}}\right]+} \\
+ & \frac{2 \pi}{J_{+}^{2}}\left[1+\frac{\chi^{2}+|w|^{2}-1}{\sqrt{\chi^{4}+2 \chi^{2}\left(|w|^{2}+1\right)+\left(|w|^{2}-1\right)^{2}}}\right] .
\end{aligned}
$$

Задачу на геодезические в $\mathrm{M}_{1}^{\chi}$ легко решить численно (см. рис. 3). Заметим, что, как и ожидалось, параллельные траектории падающих пузырей приближенно фокусируются линзой. 


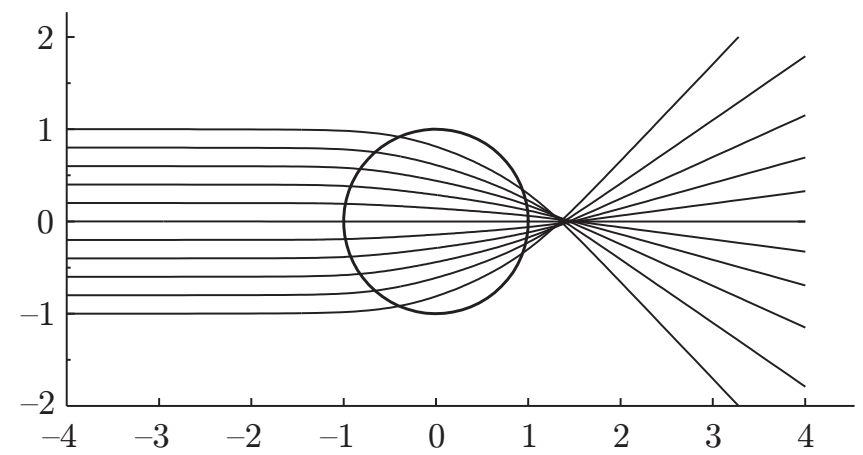

Рис. 3. Фокусировка параллельных траекторий пузырей дисковой $J$ неоднородностью. Обменный интеграл внутри диска ниже $\left(J_{-}=2\right)$, чем в окружающей плоскости $\left(J_{+}=3\right) ; \chi=0.5$.

\section{4. КВАЗИБРИЗЕРЫ}

Все рассмотренные нами $J$-неоднородности обладают некоторым типом симметрии - или трансляционной (доме́нные стенки и корыта), или вращательной (диски). В этом разделе мы по-прежнему требуем наличия вращательной инвариантности, так что $J=J(|z|)$. Однако наше внимание будет сконцентрировано совсем на другом: мы собираемся исследовать вращательно-эквивариантную динамику $n$-пузыря при $n \geqslant 2$. Это соответствует случаю $n$ совпадающих магнитных пузырей, во все моменты времени находящихся в центре симметрии $z=0$. Этот случай интересен с точки зрения динамики, потому что при $n \geqslant 2$ ширина $n$-пузыря более не является замороженным параметром. $n$-Пузырь может испытывать внутреннее вращение и расширяться или заостряться. Таким образом, интересующая нас динамика - это уже не перенос пузырей, а скорее внутреннее движение $n$-пузыря. Эту модель мы снова построим в рамках геодезической аппроксимации.

Прежде всего заметим, что если $J=J(|z|)$, то имеется естественное изометрическое действие группы $U(1)$ на $\left(\mathrm{M}_{n}, \gamma\right)$, заданное как

$$
u(z) \mapsto e^{-n i \alpha} u\left(e^{i \alpha} z\right) .
$$

Множество неподвижных точек этого действия с необходимостью является вполне геодезическим подмногообразием в $\left(\mathrm{M}_{n}, \gamma\right)$, которое мы будем называть $\left(\mathrm{M}_{n}^{\mathrm{eq}}, \gamma \mid\right)$, где $\gamma \mid$ - индуцированная метрика. Действительно [11], пространство $\mathrm{M}_{n}^{\mathrm{eq}}$ при всех $n$ диффеоморфно $\mathbb{C}^{\times}$, поскольку состоит из рациональных отображений

$$
u(z)=c z^{n}, \quad c \in \mathbb{C}^{\times} .
$$

Поскольку $U(1)$-эквивариантность является обоснованной симметрийной редукцией полного полевого уравнения (2), можно рассматривать геодезический поток в $\left(\mathrm{M}_{n}^{\mathrm{eq}}, \gamma \mid\right)$ или как низкоэнергетическую аппроксимацию симметрийно-редуцированного полевого уравнения, или как симметрийную редукцию общей геодезической аппроксимации. 
Геометрию пространства $\mathrm{M}_{n}^{\mathrm{eq}}$ легче всего понять, рассматривая подъем $\tilde{\gamma}$ метрики $\gamma \mid$ на $n$-кратное накрытие $\widetilde{\mathrm{M}}_{n}^{\mathrm{eq}}$ пространства $\mathrm{M}_{n}^{\mathrm{eq}}$ [11]. Это пространство снова диффеоморфно $\mathbb{C}^{\times}$, а проекция накрытия имеет вид

$$
\pi: \widetilde{\mathrm{M}}_{n}^{\mathrm{eq}} \rightarrow \mathrm{M}_{n}^{\mathrm{eq}}, \quad \pi(\rho)=\rho^{-n} .
$$

Поэтому $n$-пузырь, соответствующий $\rho \in \mathbb{C}^{\times}$, есть $u(z)=(z / \rho)^{n}$. Ясно, что любая из $n$ точек $\rho e^{2 \pi i k / n}$ в $\widetilde{\mathrm{M}}_{n}^{\mathrm{eq}}, k=0,1, \ldots, n-1$, дает тот же $n$-пузырь. Можно думать о $|\rho|$ как о ширине $n$-пузыря, а аргумент $\rho$ по модулю $2 \pi / n$ считать внутренней фазой. Плотность потенциальной энергии $n$-пузыря обладает вращательной симметрией, обращается в нуль при $z=0$ и $|z| \rightarrow \infty$ и достигает максимума при

$$
|z|=|\rho|\left(\frac{n-1}{n+1}\right)^{1 / 2 n}
$$

Поднятая метрика на $\widetilde{\mathrm{M}}_{n}^{\mathrm{eq}}$ имеет вид

$$
\tilde{\gamma}=f(|\rho|) d \rho d \bar{\rho}, \quad f(|\rho|)=8 \pi n^{2} \int_{0}^{\infty} d r \frac{r^{2 n+1}}{\left(1+r^{2 n}\right)^{2} J(|\rho| r)^{2}}
$$

В однородном случае $J(|z|)=J_{0}$ (константа) метрика имеет вид $\tilde{\gamma}=C_{n} d \rho d \bar{\rho}$, где $C_{n}$ - константа, зависящая от $n$, так что $\left(\widetilde{\mathrm{M}}_{n}^{\mathrm{eq}}, \tilde{\gamma}\right)$ в действительности изометрична евклидовой проколотой плоскости, и поднятые геодезические являются просто прямыми линиями, пересекаемыми при постоянной скорости. Для всех геодезических $|\rho| \rightarrow \infty$ как при $t \rightarrow \infty$, так и при $t \rightarrow-\infty$, за исключением случая, когда $\rho(t)$ представляет собой луч, попадающий в выколотую точку $\rho=0$ в обеих ситуациях. Лучи (если они направлены внутрь) соответствуют картине, когда $n$-пузырь с нулевым моментом импульса за конечное время коллапсирует с образованием сингулярности. Прямые линии, не попадающие в $\rho=0$, соответствуют $n$-пузырям, обладающим моментом импульса, неограниченно расширяющимся при $|t| \rightarrow \infty$. В итоге каждый спин $\mathbf{n}(z)$ в таком пузыре совершает $n / 2$ полных вращений вокруг оси $n_{3}$.

Несколько более интересное поведение получается, если разрешить $J(|z|)$ не быть постоянной величиной. "Сферический” случай $J(|z|)=1+|z|^{2}$ подробно изучался в работе [11]. Приятный и достаточно неожиданный факт в этом случае состоит в том, что объем пространства $\mathrm{M}_{n}^{\mathrm{eq}}$, который оказывается конечным, в действительности не зависит от $n$. Можно изометрически вложить $\mathrm{M}_{n}^{\mathrm{eq}}$ в $\mathbb{R}^{3}$ как поверхность вращения, причем эта поверхность будет иметь конические сингулярности с угловым дефицитом $2 \pi\left(1-n^{-1}\right)$ при $c=0$ и $c=\infty$. Однако в этом случае значение $J$ не ограничено, что неприемлемо с физической точки зрения. Мы рассмотрим случай круглой линзы, когда значение $J$ внутри диска с центром в начале координат меньше, чем его значение вне диска. Для простоты используем предел резкой стенки, т.е.

$$
J(|z|)= \begin{cases}J_{+}, & |z|>1 \\ J_{-}, & |z| \leqslant 1\end{cases}
$$


где $J_{+}>J_{-}$. В предыдущем разделе мы видели, что для такого $J(|z|)$ метрику на $\mathrm{M}_{1}^{\chi}$ можно посчитать явно. Подчеркнем, однако, что это не та метрика, которая нас сейчас интересует. Напомним, что координаты в пространстве $\mathrm{M}_{1}^{\chi}$ задаются координатами пузырей на физической плоскости, а ширина пузыря $\chi$ является замороженным параметром. Напротив, координаты в пространстве $\mathrm{M}_{n}^{\text {eq }}$ задаются шириной $n$-пузыря и внутренней фазой, а координаты на физической плоскости при этом фиксированы. В работе [2] в геодезическом потоке в пространстве $\mathrm{M}_{1}^{\chi}$ при изменении $\chi$ наблюдалась интересная бифуркация: при возрастании $\chi$ выше критического значения появлялась пара периодических геодезических, вдоль которых бугор вращается по окружности с центром в $z=0$, - одна геодезическая внутри линзы, другая вне ее. Как мы увидим, в геодезическом потоке на $\widetilde{\mathrm{M}}_{n}^{\mathrm{eq}}$ имеется геометрически похожая бифуркация, но ее физический смысл совершенно иной, и параметром бифуркации является отношение $J_{+} / J_{-}$, а не ширина пузыря (которая стала динамической переменной).

Полагая, что $J$ имеет вид (29), получаем

$$
f(|\rho|)=\frac{2 \pi n^{2}}{J_{-}^{2}}\left\{\frac{\pi}{n^{2} \sin (\pi / n)}-\left(1-\frac{J_{-}^{2}}{J_{+}^{2}}\right) \int_{0}^{|\rho|^{2}} d s \frac{s^{n-2}}{\left(1+s^{n}\right)^{2}}\right\} .
$$

Поскольку подынтегральное выражение рационально, можно в принципе явно записать $f$ для любого выбора $n$. Однако получающиеся выражения даже при малых $n$ не слишком много проясняют, так что мы их опустим. Более полезным для нас является тот факт, что если отношение $J_{+} / J_{-}$не слишком велико, то можно изометрически вложить $\widetilde{\mathrm{M}}_{n}^{\text {eq }}$ как поверхность вращения в $\mathbb{R}^{3}$. Образующие кривые этих поверхностей в случае $n=2$, порожденном методом, описанным в работе [11], представлены на рис. 4. Если $J_{+} / J_{-}=1$, то поверхность представляет собой просто (проколотую) плоскость, заметаемую вращающейся полупрямой. При увеличении отношения $J_{+} / J_{-}$образующая кривая принимает форму бугра, пока наконец над суженной горловиной не образуется горб. Здесь происходит бифуркация геодезического потока. Появляется пара периодических геодезических с постоянным значением $|\rho|$, и имеются квазипериодические (и периодические) геодезические, которые остаются заключенными внутри горба. Они соответствуют квазибризерам. При дальнейшем возрастании отношения $J_{+} / J_{-}$изометрическое вложение теряется, но существование квазибризерных геодезических сохраняется.

Для подтверждения этой наглядной аргументации снова рассмотрим фазовый портрет геодезического потока. Вводя полярные координаты $(\sigma, \psi)$ на $\widetilde{\mathrm{M}}_{n}^{\mathrm{eq}}$ с помощью формулы $\sigma e^{i \psi}:=\rho$, получаем, что поток порождается гамильтонианом

$$
H=\frac{1}{2 f(\sigma)}\left(p_{\sigma}^{2}+\frac{p_{\psi}^{2}}{\sigma^{2}}\right)
$$

с сопряженными импульсами

$$
p_{\sigma}=f(\sigma) \dot{\sigma}, \quad p_{\psi}=\sigma^{2} f(\sigma) \dot{\psi} .
$$

Как $H$, так и $p_{\psi}$ являются интегралами движения, и без потери общности можно выбрать $H=1 / 2$. Траектории после проекции на полуплоскость $\left(\sigma, p_{\sigma}\right)$ (заметим, 


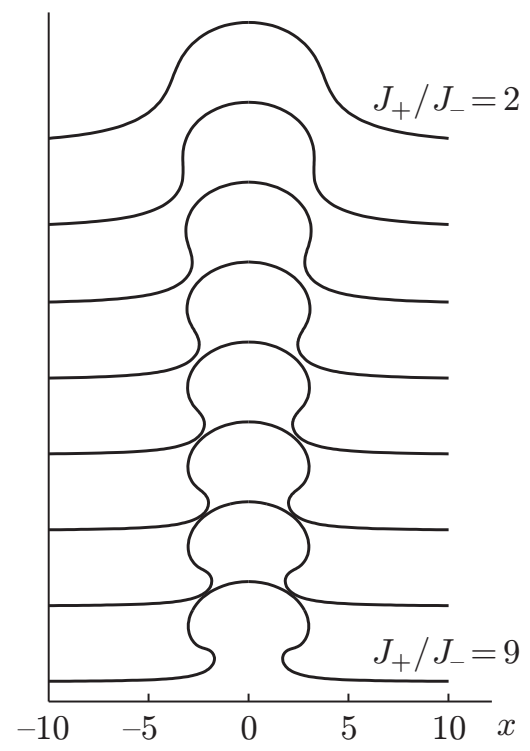

Рис. 4. Образующие для $\widetilde{\mathrm{M}}_{2}^{\mathrm{eq}}$, изометрически вложенной как поверхность вращения в $\mathbb{R}^{3}$, в случае резкой дисковой $J$-неоднородности при $J_{-} / J_{+}=$ $2,3, \ldots, 9$ (сверху вниз). Поверхности, заметаемые этими кривыми, вращаются вокруг вертикальной оси $x=0$. Заметим, что горловина развивается при увеличении отношения $J_{+} / J_{-}$.

что $\sigma>0)$ задаются множествами постоянного уровня

$$
\sigma^{2}\left(f(\sigma)-p_{\sigma}^{2}\right)=p_{\psi}^{2}
$$

где $p_{\psi}^{2} \in[0, \infty)$. Эти множества постоянного уровня для $J_{+} / J_{-}=2$ и $J_{+} / J_{-}=4$ в случае $n=2$ показаны на рис. 5 . Заметим, что когда отношение $J_{+} / J_{-}$превосходит критическое значение (около 2.89 при $n=2$ ), на фазовом портрете появляется островок из периодических орбит. Это происходит из-за образования локального максимума величины $\sigma^{2} f(\sigma)$, а также, с учетом асимптотичского поведения $\sigma^{2} f(\sigma) \sim 4 \pi J_{+}^{-2} \sigma^{2}$ при $\sigma \rightarrow \infty$, из-за образования пары критических точек $\sigma^{2} f(\sigma)$.

$\mathrm{У}$ этих точек есть изящная геометрическая интерпретация, которую мы сейчас опишем. Во-первых, отметим, что

$$
\frac{d}{d \sigma} \sigma^{2} f(\sigma)=0 \quad \Leftrightarrow \quad \Xi(\sigma):=1+\frac{\sigma f^{\prime}(\sigma)}{2 f(\sigma)}=0 .
$$

Теперь необходимое и достаточное условие того, чтобы пространство $\widetilde{\mathrm{M}}_{n}^{\mathrm{eq}}$ изометрически вкладывалось как поверхность вращения в $\mathbb{R}^{3}$, имеет вид $-1 \leqslant \Xi(\sigma) \leqslant 1$ для любых $\sigma$ (см. [11]). В этом случае, если мы представим себе поверхность, порожденную вращением производящей кривой в плоскости $x_{1} x_{2}$ вокруг оси $x_{3}$ (как показано на рис. 4$)$, то $\Xi(\sigma)=\cos \xi(\sigma)$, где $\xi$ - угол между касательной к производящей 

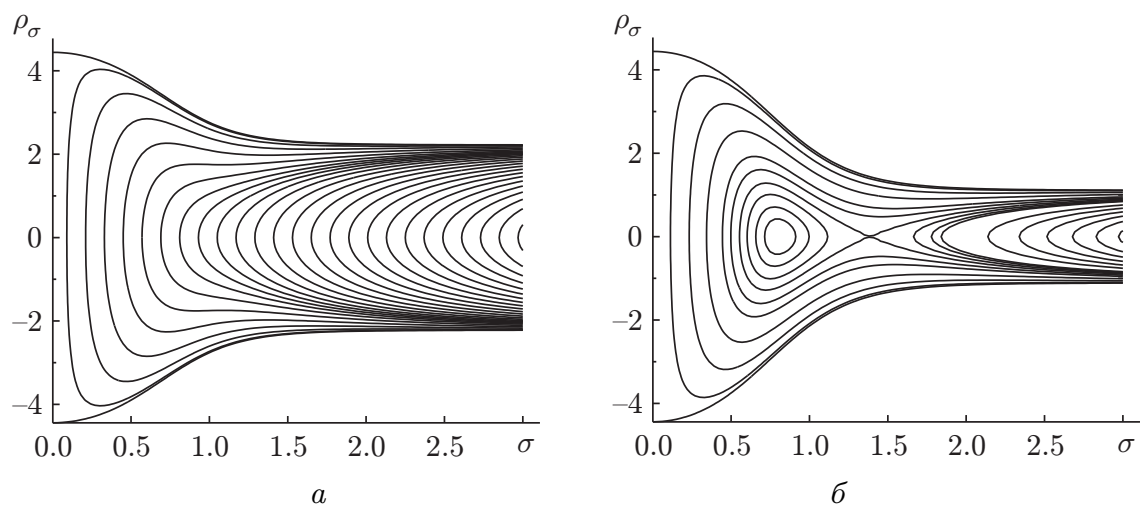

Рис. 5. Фазовые портреты геодезического потока в $\widetilde{\mathrm{M}}_{2}^{\mathrm{eq}}$ для $J_{+} / J-=2$ (а) и $J_{+} / J_{-}=4$ (б). Заметим, что при больших значениях отношения $J_{+} / J_{-}$ образуется островок периодических орбит.

кривой и осью $x_{1}$. Таким образом, критические точки $\sigma^{2} f(\sigma)$ связаны с точками, где касательная к производящей кривой вертикальна, и пара таких точек развивается в точности тогда, когда поверхность развивается в суженную горловину. Это подтверждает наши наглядные соображения относительно того, что островок замкнутых траекторий появляется, когда в $\widetilde{\mathrm{M}}_{n}^{\text {eq }}$ образуется суженная горловина.

Напомним, что траектории, изображенные на рис. 5, являются проекциями геодезических на плоскость $\left(\sigma, p_{\sigma}\right)$. Замкнутая траектория на этом рисунке не обязательно соответствуют периодической геодезической: как ширина $\sigma(t)$, так и фаза $e^{i \psi(t)}$ являются периодическими, но с (вообще говоря) несоизмеримыми периодами. Таким образом, эти геодезические являются только квазипериодическими, поэтому мы называем связанные с ними осциллирующие $n$-пузыри квазибризерами. Временны́ зависимости ширины $\sigma$ и внутренней фазы $\psi$ типичного квазибризера изображены на рис. 6. Заметим, что форма этих решений действительно "дышит". Это противоречит внутреннему вращению магнитных солитонов (иногда называемых бризерами), которое возникает в ферромагнитных спиновых решетках (с оnsite анизотропией), где $n_{3}$ имеет фиксированный профиль в форме бугра, а спины только вращаются с постоянной скоростью вокруг оси $n_{3}$ [12]. При $n=2$ квазибризеры существуют только при $J_{+} / J_{-}>2.89$ (приближенно), но при любом значении $J_{+} / J_{-}>1$ имеется некоторое $n_{\min } \geqslant 2$ такое, что геодезический поток поддерживает квазибризеры любой степени $n \geqslant n_{\min }$. Зависимость $n_{\min }$ от отношения $J_{+} / J_{-}$ показана на рис. 7.

\section{5. ЗАКЛЮЧИТЕЛЬНЫЕ ЗАМЕЧАНИЯ}

Мы показали, что в почти континуальном режиме двумерный изотропный антиферромагнетик Гейзенберга с зависящим от координат обменным интегралом описывается релятивистской $O(3)$ сигма-моделью на пространстве-времени с пространственными неоднородностями. В силу конформной инвариантности такая модель 


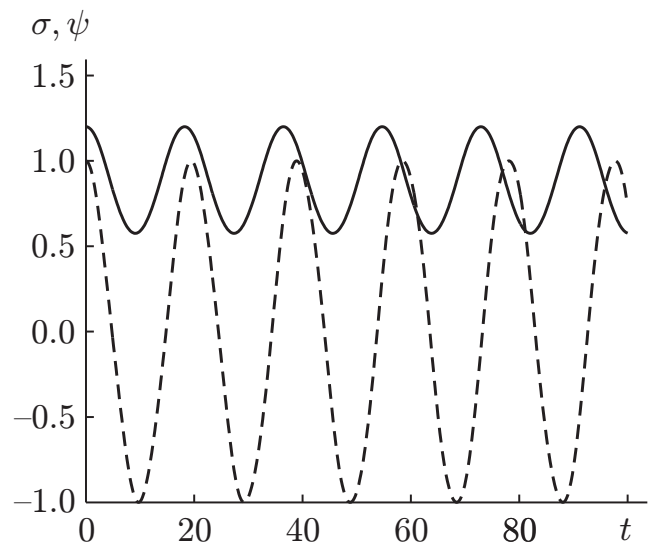

Рис. 6. Времень́е зависимости ширины $\sigma$ (сплошная линия) и внутренней фазы $\psi$ (штриховая линия) типичного квазибризера степени 2 для дисковой неоднородности с $J_{+} / J_{-}=4$.

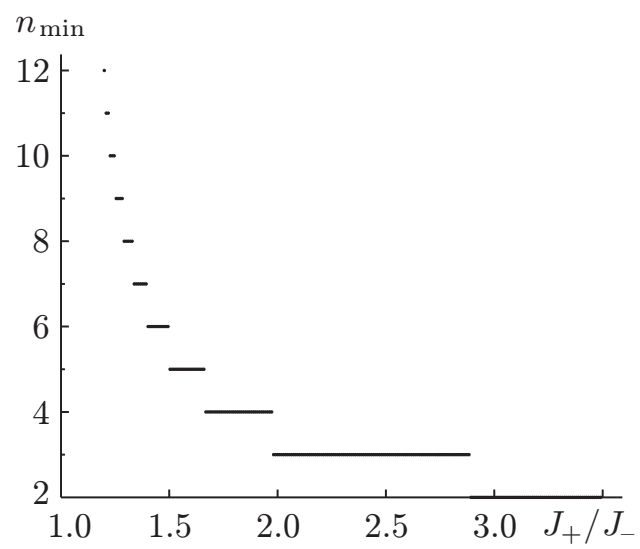

Рис. 7. Минимально разрешенная степень $n_{\min }$ квазибризера как функция параметра неоднородности $J_{+} / J_{-}$.

поддерживает статические бугры Белавина-Полякова, которые мы называем пузырями. Предсказывается, что траектории этих пузырей ведут себя как световые лучи, распространяющиеся в неоднородной среде с отождествляемым с показателем преломления обратным обменным интегралом $J^{-1}$. В частности, пузыри, падающие на прямую доме́нную стенку, преломляются в соответствии с законом Снеллиуса, при этом может происходить полное внутреннее отражение. Такое явление преломления было отмечено в работе [2].

В настоящей работе мы дали обзор динамики пузырей, исследованной в работе [2], и распространили этот анализ за пределы круга изучавшихся ранее задач рассеяния, 
включив в рассмотрение вращательно-эквивариантную динамику пузырей высших степеней. Оказалось, что при достаточно больших значениях отношения $J_{+} / J_{-}$ дисковые $J$-неоднородности поддерживают квазибризеры, т.е. пузыри степени $n \geqslant 2$ с центрами на диске, которые испытывают внутреннее вращение, в то время как их форма осциллирует с несоизмеримыми в случае общего положения периодами.

Наш анализ был проведен целиком в рамках геодезической аппроксимации Мантона. Обоснованность этой аппроксимации для $O(3)$ сигма-модели остается открытым вопросом. В общем контексте релятивистских теорий поля типа теорий Богомольного было доказано, что эта аппроксимация весьма успешно описывает процессы рассеяния солитонов при энергиях от низких до средних, когда солитоны движутся в основном (кроме относительно короткой фазы взаимодействия) как свободные частицы [9]. Поэтому основной эффект преломления на доме́нной стенке напоминает устойчивую картину динамики пузыря степени 1.

Статус квазибризерных решений не такой простой. Маловероятно, что сигмамодель будет поддерживать истинные квазипериодические решения для всякого ограниченного выбора $J(|z|)$, поскольку следует ожидать, что осциллирующий вращающийся пузырь будет излучать энергию на бесконечность в виде малых распространяющихся волн. Поэтому полученные в настоящей работе квазипериодические геодезические в пространстве $\widetilde{\mathrm{M}}_{n}^{\mathrm{eq}}$ не могут глобально быть близки к истинным решениям модели с теми же начальными условиями. Геодезическую аппроксимацию следует рассматривать как способ предсказания того, что в модели существуют длительно осциллирующие вращающиеся пузыри, которые приближенно являются квазипериодическими, но распадаются на долговременном масштабе.

Единственные геодезические, для которых были получены строгие оценки ошибок, - это радиальные лучи $\rho(t)=-v t$ в пространстве $\widetilde{\mathrm{M}}_{n}^{\text {еq }}$ для $n \geqslant 3$ (в однородном случае, когда значение $J$ постоянно, хотя это и не обязательно является решающим моментом). Эти геодезические описывают эквивариантный $n$-пузырь, коллапсирующий за конечное время. В работе [13] было показано, что на самом деле решения действительно коллапсируют за конечное время, но при этом к скорости коллапса, предсказанной геодезической аппроксимацией, добавляется логарифмическая поправка, которая становится значительной, когда пузырь совсем близок к коллапсу (т.е. становится совсем узким). В нашем контексте значение этого отклонения является спорным: когда пузырь очень узкий, континуальная аппроксимация, ведущая к сигма-модели, уже нарушена. Действительно, наиболее важная проверка динамических явлений, предсказанных в настоящей работе и в работе [2], проводится не с помощью строгого анализа ДУЧП (2), а прямым численным моделированием самой спиновой решетки (1). С точки зрения вычислений такие численные расчеты требуют гораздо больше усилий, чем методы, которые мы использовали в настоящей работе, и выходят за ее рамки.

Благодарности. Настоящая работа в значительной степени основана на докладе "Преломление магнитных пузырей в неоднородных антиферромагнетиках," сделанном автором на конференции "Нелинейная физика. Теория и эксперимент IV". Участие автора в этом совещании финансировалось EPSRC. 


\section{Список литературы}

[1] F. D. M. Haldane, Phys. Lett. A, 93 (1983), 464.

[2] J. M. Speight, Nonlinearity, 19 (2006), 1565.

[3] S. Komineas, N. Papanicolaou, Nonlinearity, 11 (1998), 265.

[4] А. А. Белавин, А. М. Поляков, Писъма в ЖЭТФ, 22 (1975), 503.

[5] N.S. Manton, Phys. Lett. B, 110 (1982), 54.

[6] R. S. Ward, Phys. Lett. B, 158 (1985), 424.

[7] R. A. Leese, Nucl. Phys. B, 344 (1990), 33.

[8] P. J. Ruback, Nucl. Phys. B, 296 (1988), 669.

[9] N.S. Manton, P. M. Sutcliffe, Topological Solitons, Cambridge Univ. Press, Cambridge, 2004.

[10] J. M. Speight, J. Math. Phys., 36 (1995), 796.

[11] J. A. McGlade, J. M. Speight, Nonlinearity, 19 (2006), 441.

[12] A. M. Kosevich, B. A. Ivanov, A. S. Kovalev, Phys. Rep., 194 (1990), 117.

[13] I. Rodnianski, J. Sterbenz, On the formation of singularities in the critical O(3) sigmamodel, math.AP/0605023. 